\title{
Outage and Coverage Considerations for Microcellular Mobile Radio Systems in a Shadowed-Rician/Shadowed-Nakagami Environment
}

\author{
Jen-Cheng Lin, Wen-Chang Kao, Yu T. Su, Member, IEEE, and Tsern-Huei Lee, Member, IEEE
}

\begin{abstract}
This paper presents outage analysis and the associated reuse distance and service area estimations for microcellular mobile radio systems that operate in a shadowedRician/shadowed-Nakagami fading environment. Outages caused either by the minimum carrier-to-interference ratio (CIR) requirement alone or by the CIR plus the minimum signal strength [or, equivalently, carrier-to-noise ratio $(\mathrm{CNR})]$ requirements are considered. The desired signal is assumed to suffer from Rician fading while the interference signals from cochannel cells experience Nakagami fading. The interferers may have identical or different statistics. In addition, the local mean strength of the desired and interference signals may fluctuate due to shadowing.
\end{abstract}

Index Terms-Coverage, microcellular, Nakagami, outage, Rician.

\section{INTRODUCTION}

$\mathbf{F}$ REQUENCY reuse is the core concept for increasing the spectrum efficiency of a cellular radio system. The limitation and effect of simultaneous use of the same frequency in different cells can be assessed through the evaluation of the outage probability, that is, the probability that the carrierto-interference ratio (CIR) for a received signal is less than a minimum required value. Of course, when carrier-to-noise ratio (CNR) does not exceed a threshold value, outage can also occur. However, for a microcellular personal communication system that has a short frequency reuse distance, outage is expected to be caused primarily by cochannel interference (CCI). Outage is closely related to the reuse distance and the coverage (service) area of a cellular system. The reuse distance is the minimum distance between any two cochannel base stations which ensures a worst case outage probability no larger than the required value and the coverage area refers to the area within which the outage probability is guaranteed to be less than a given threshold. In determining the reuse distance, one usually assumes regular-shaped communication cells and uniform unobstructed terrain characteristics. On the other hand, in predicting the coverage area of a base station, one has to know at least the real two-dimensional (2-D) road

Manuscript received July 7, 1996; revised February 21, 1997. This paper was presented in part in December 1996 at the National Telecommunication Symposium on Telecommunications, Chung-Li, Taiwan, R.O.C.

J.-C. Lin and W.-C. Kao were with the Department of Communication Engineering, National Chiao Tung University, Hsinchu, Taiwan, R.O.C. They are now with the National Space Program Office, Taiwan.

Y. T. Su and T.-H. Lee are with the Department of Communication Engineering, National Chiao Tung University, Hsinchu, Taiwan, R.O.C.

Publisher Item Identifier S 0018-9545(99)00680-5. topology, the reuse pattern and the locations of the neighboring base stations.

The statistical properties of a communication environment are often characterized by three propagation effects: 1) shortterm (small area) fading; 2) long-term (large area) shadowing; and 3) propagation path loss. For a street microcellular system with base station antenna height at street-lamp level, the received signal from a line-of-sight (LOS) path usually contains a direct nonfading component. The statistic of this LOS signal's envelope tends to follow a Rician distribution [1]. Interference signals from cochannel cells usually lack a direct path component at the receiving location, and the Nakagami distribution (i.e., $m$ distribution) [2] is versatile in characterizing the statistics of their envelopes [3]-[5]. Nakagami distribution can be reduced to Rayleigh distribution and can model fading conditions more or less severe than those in the Rayleigh case. With proper chosen parameters it can also fit Rician and lognormal distributions very tightly. Many other fading models such as Rician/Rayleigh [6], Rician/Rician [7]-[9], Nakagami/Nakagami [4], [5], and Rayleigh/Rician [10] models have been proposed to investigate the impact of CCI on the performance of a cellular system. In addition to the short-term fading, many experiments show that a mobile signal's local mean power fluctuates and its statistical property, in both conventional cellular [11], [12] and urban microcellular environments [13], [14], can be described by a lognormal distribution.

The purpose of this paper is to derive outage probabilities and estimate the associated reuse distance and coverage area of microcellular radio systems in a shadowed-Rician/shadowedNakagami fading environment. Section II contains statistical descriptions of the short-term and long-term fading (shadowing) models. The probability density function (pdf) of the desired signal power and those of single and multiple interferers (with identical or different parameters) are provided. Section III presents our outage analysis for a variety of microcellular environments. We obtain closed-form expressions for the outage probabilities caused by the minimum CIR plus the minimum signal-to-noise ratio (SNR) requirements. CIRinduced outage is an important special case for which compact closed-form formulas are also given. The effect of combined fading and shadowing are analyzed subsequently. Using the outage probability analysis results, we then evaluate the reuse distance and determine the service area in Section IV. The difference between fixed and random number of interferers 
is examined. Section V presents numerical results and related discussion. The final section summarizes our main results and presents conclusions.

\section{COMMUNICATION ENVIRONMENT}

\section{A. Local Statistic of the Desired Signal}

In a Rician faded channel, the envelope of the desired signal $\beta_{s}$ is distributed according to the Rician pdf

$$
f\left(\beta_{s}\right)=\frac{\beta_{s}}{\tilde{x}} \exp \left(-\frac{\beta_{s}^{2}+S^{2}}{2 \tilde{x}}\right) I_{0}\left(\frac{\beta_{s} S}{\tilde{x}}\right)
$$

where $I_{0}(\cdot)$ is the zeroth-order modified Bessel function of the first kind. The pdf of the corresponding signal power $x=\beta_{s}^{2} / 2$ is given by

$$
f_{X}(x)=\frac{1}{\tilde{x}} \exp \left(-\frac{2 x+S^{2}}{2 \tilde{x}}\right) I_{0}\left(\frac{\sqrt{2 x} S}{\tilde{x}}\right) .
$$

In (1) and (2), $\widetilde{x}$ is the mean power of the diffuse components and $S^{2} / 2$ is the mean power of the specular component, hence, the total local mean power is $\bar{x}=\tilde{x}+S^{2} / 2$. The Rician distribution is characterized by the Rician factor $a$, which is defined as the ratio of the mean specular power to the mean diffuse power, i.e., $K=S^{2} /(2 \tilde{x})$. Measurement data show that the Rician factor in a microcellular environment typically falls in the range of $7-12 \mathrm{~dB}$ with $7 \mathrm{~dB}(K=5)$ being a typical value [1]. With the definition of Rician factor, (2) can be rewritten as

$$
\begin{aligned}
f_{X}(x)= & \frac{K+1}{\bar{x}} \exp \left(-K-\frac{(K+1) x}{\bar{x}}\right) \\
& \times I_{0}\left(\sqrt{\frac{4 K(K+1)}{\bar{x}} x}\right) .
\end{aligned}
$$

\section{B. Local Statistic of an Interference Signal}

The envelope of an interferer $\beta_{I}$ is assumed to follow a Nakagami pdf

$$
g\left(\beta_{I}\right)=\frac{2}{\Gamma(m)}\left(\frac{m}{2 \bar{y}}\right)^{m} \beta_{I}^{2 m-1} \exp \left(-\frac{m}{2 \bar{y}} \beta_{I}^{2}\right)
$$

where $\Gamma(\cdot)$ is the Gamma function, $\bar{y}$ is the local mean interference power, and $m$ is the fading parameter. The degree of fading is characterized by $1 / \mathrm{m}$, the ratio of the variance of the received energy to the square of the mean received energy [2]. As $m$ becomes smaller, the degree of fading becomes severer. The Rayleigh distribution and the one-sided Gaussian distribution can be obtained by setting $m=1$ and $m=1 / 2$ in (4), respectively. The fading parameter $m$ can be any real value greater than or equal to $1 / 2$, but we will consider cases of integer values of $m$ only. Our numerical study indicates that performance for cases with noninteger fading parameters can be tightly bounded by those of integer fading parameters [15]. The pdf of the interference power $y=\beta_{I}^{2} / 2$ for integer-valued $m$ is then given by

$$
g_{Y}(y)=\left(\frac{m}{\bar{y}}\right)^{m} \frac{y^{m-1}}{\Gamma(m)} \exp \left(-\frac{m}{\bar{y}} y\right)
$$

In the case that there are $L$ mutually independent Nakagami interferers, the total interference power $y$ is given by

$$
y=y_{1}+y_{2}+\cdots+y_{L}
$$

where $y_{i}$ is a random variable representing the local (shortterm) power of the $i$ th interferer. The pdf of $y$ is [4]

$$
\begin{aligned}
g_{Y}(y)= & {\left[\prod_{k=1}^{L}\left(\frac{m_{k}}{\bar{y}_{k}}\right)^{m_{k}}\right] } \\
& \times \sum_{k=1}^{L} \exp \left(-\frac{m_{k}}{\bar{y}_{k}} y\right) \sum_{i=1}^{m_{k}} \frac{z_{k}^{i-1} y^{m_{k}-i}}{\left(m_{k}-i\right) !(i-1) !}
\end{aligned}
$$

where $\bar{y}_{k}$ and $m_{k}$ are the local mean power and the fading parameter for the $k$ th interferer, respectively, and

$$
z_{k}^{i-1}=\left.\frac{d^{i-1}}{d s^{i-1}}\left[\prod_{\substack{\nu=1 \\ \nu \neq k}}^{L}\left(\frac{m_{\nu}}{\bar{y}_{\nu}}+s\right)^{-m_{\nu}}\right]\right|_{s=-m_{k} / \bar{y}_{k}} .
$$

When $L=1$ or $m_{1} / \bar{y}_{1}=m_{2} / \bar{y}_{2}=\cdots=m_{L} / \bar{y}_{L}=m / \bar{y}$, (7) is no longer valid. In this case, the pdf of the resultant interference power $y$ is given by

$$
g_{Y}(y)=\left(\frac{m}{\bar{y}}\right)^{m_{t}} \frac{y^{m_{t}-1}}{\Gamma\left(m_{t}\right)} \exp \left(-\frac{m}{\bar{y}} y\right)
$$

where $m_{t}=\sum_{k=1}^{L} m_{k}$.

\section{OUtAgE ANALYSIS}

We begin our analysis by considering outage probabilities due to both cochannel interference and thermal noise. Various CCI-induced outage probabilities are presented, and we also discuss the effects of fading and shadowing.

\section{A. Outage Due to CCI and Thermal Noise}

Let $x, y$ be the strengths of the desired and the undesired signals. Since both minimum CIR and minimum desired signal strength requirements have to be met, the outage probability is given by

$$
\begin{aligned}
P_{\text {out }}(L ; I, N) & =1-\operatorname{Pr}\left(x>\gamma_{0}, x / y>q\right) \\
& =1-\int_{\gamma_{0}}^{\infty} f_{X}(x) \int_{0}^{x / q} g_{Y}(y) d y d x
\end{aligned}
$$

where $L$ is the number of interferers, $I$ is used to indicate the presence of CCI, and $N$ emphasizes the influence of thermal noise. $\gamma_{0}$ is the required minimum signal power, and $q$ is the system protection ratio (minimum CIR required). Practical values for $q$ are $18 \mathrm{~dB}$ for analog FM systems and $20 \mathrm{~dB}$ for linear SSB modulation systems [17]. Other typical values of $q$ used before lie between 7-20 dB.

To present a compact closed-form expression for the outage probability $P_{\text {out }}(L ; I, N)$, we need the following definitions: $c=(K+1) / \xi, \xi=\bar{x} / \gamma_{0}, w_{k}=\left(m_{k} b_{k} / q(K+1)\right)$, and $b_{k}=\bar{x} / \bar{y}_{k} . b_{k}$ is the local mean power ratio between the desired signal and the $k$ th interferer. $\xi$ is the power ratio of the 
desired to the required minimum. When measured in decibels, $\xi$ represents the excess power of the desired signal over the minimum required. Using the above parameters we can show (see Appendix I-B)

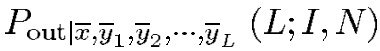

$$
\begin{aligned}
& =1-\exp (-K-c) \\
& \times\left(\prod_{k=1}^{L} w_{k}^{m_{k}}\right) \sum_{k=1}^{L} \sum_{i=1}^{m_{k}} \frac{D_{k}^{i-1}}{(i-1) !} w_{k}^{-m_{k}+i-1} \\
& \times\left\{\sum_{h=0}^{\infty} \sum_{l=0}^{h} \frac{K^{h}}{h !} \frac{d^{l}}{l !}-\exp \left(-c w_{k}\right)\right. \\
& \left.\times \sum_{h=0}^{\infty} \sum_{j=0}^{m_{k}-i} \sum_{n=0}^{j+h} \frac{(j+h) !}{j ! h ! h ! n !} \frac{K^{h} c^{n} w_{k}{ }^{j}}{\left(1+w_{k}\right)^{j+h+1-n}}\right\}
\end{aligned}
$$

where

$$
D_{k}^{i-1}=\left.\frac{d^{i-1}}{d s^{i-1}}\left[\prod_{\substack{\nu=1 \\ \nu \neq k}}^{L}\left(w_{\nu}+s\right)^{-m_{\nu}}\right]\right|_{s=-w_{k}} .
$$

The above equation represents the case when the received interference signals, having travelled through different paths (with unequal lengths) and environments, yield different mean powers and fading parameters. When all interferers have identical statistics, (11) becomes (see Appendix I-A)

$$
\begin{aligned}
P_{\text {out } \mid \bar{x}, \bar{y}}(L ; I, N) & \\
= & 1-\exp (-K-c) \sum_{h=0}^{\infty} \sum_{i=0}^{h} \frac{K^{h} c^{i}}{h ! i !} \\
+ & \exp (-K-c(1+w)) \sum_{h=0}^{\infty} \sum_{k=0}^{m L-1} \sum_{n=0}^{k+h} \\
& \cdot\left\{\frac{(k+h) !}{k ! h ! h ! n !} \times \frac{K^{h} c^{n} w^{k}}{(1+w)^{k+h+1-n}}\right\}
\end{aligned}
$$

where $w=(m b / q(K+1))$ and $b=\bar{x} / \bar{y}$. If $L=1$ or if every interferer has the same $m_{i} / \bar{y}_{i}$, then $w_{i}$ becomes independent of $i$. Denoting the common value for all $w_{i}$ by $w_{0}$, one can show that the associated outage probability is identical to (13) with $m L=m_{t}$ and $w=w_{0}$.

Other cases like interferers with identical fading parameters, but unequal mean powers or equal mean power, but different fading parameters can be derived from the above equations. There is, however, a set of cases of particular interest in which more compact expressions for the associated outage probabilities can be derived in an alternative way, namely, the CCI-limited operation environment. For this special case, outage occurs only if CIR is less than the system protection ratio. The outage probability for this case is denoted by $P_{\text {out }}(L ; I)$, where the parameter $N$ has been dropped since thermal noise is ignored. When $L$ independent and identically distributed (i.i.d.) interferers are present, $P_{\text {out }}(L ; I)$ can be obtained by first substituting (3) and (9) with $m_{t}=m L, \gamma_{0}=$
0 into (10) and using [18, eqs. (3.381.3) and (6.643.2)]. The resulting expression is

$$
\begin{aligned}
P_{\text {out } \mid \bar{x}, \bar{y}}(L ; I)= & \exp \left(\frac{-K w}{1+w}\right) \sum_{k=0}^{m L-1} \frac{w^{k}}{(1+w)^{k+1}} \\
& \times \sum_{i=0}^{k}\left(\begin{array}{c}
k \\
i
\end{array}\right) \frac{1}{i !}\left(\frac{K}{1+w}\right)^{i}
\end{aligned}
$$

where $\left(\begin{array}{c}k \\ i\end{array}\right)=(k ! /(k-i) ! i !)$. In the case that the $L$ independent interferers have different statistics, the outage probability can be obtained by substituting (3) and (7) into (10) and set $\gamma_{0}$ to zero. The corresponding outage probability is

$$
\begin{aligned}
P_{\mathrm{out} \mid} \mid \bar{x}, \bar{y}_{1}, \bar{y}_{2}, \cdots, \bar{y}_{L}(L ; I) & \\
= & \left(\prod_{i=k}^{L} w_{k}^{m_{k}}\right) \\
& \times \sum_{k=1}^{L} \exp \left(-\frac{K w_{k}}{1+w_{k}}\right) \sum_{i=1}^{m_{k}} \frac{D_{k}^{i-1}}{(i-1) !} w_{k}^{-m_{k}+i-1} \\
& \times \sum_{j=0}^{m_{k}-i} \frac{w_{k}^{j}}{\left(1+w_{k}\right)^{j+1}} \sum_{n=0}^{j}\left(\begin{array}{l}
j \\
n
\end{array}\right) \frac{1}{n !}\left(\frac{K}{1+w_{k}}\right)^{n} .
\end{aligned}
$$

Equation (15) can be used to calculate outage probabilities for the case of $L(\geq 2)$ interferers with unequal mean power and different fading parameters, provided that $m_{\nu} b_{\nu} \neq m_{k} b_{k}$ for $\nu \neq k$. Equations (14) and (15) are derived in Appendix II. When $L=1$ or $m_{\nu} / \bar{y}_{\nu}=m_{k} / \bar{y}_{k}$ for $\nu \neq k$, (15) is no longer valid. After substituting (3) and (9) and $\gamma_{0}=$ 0 into (10), replacing the modified Bessel function by its series representation (I.3), and using the identity (I.4), we conclude that the outage probability for this special case can be evaluated by (14) with the substitutions $m L=m_{t}$ and $w=w_{0}$.

\section{B. Effect of Shadowing}

As a mobile unit moves around, the received field strength varies. Experiments have indicated [13], [14] that the local mean power follows a lognormal distribution. If we assume that the propagating paths are uncorrelated and the traveled topography or the propagation environments are analogous so that the statistic of each incoming waveform is independent and has the same standard deviation $\sigma$, then the local mean powers of the desired $\bar{x}$ and those undesired $\bar{y}_{1}, \bar{y}_{2}, \cdots, \bar{y}_{L}$ follow a joint lognormal distribution, as given in (16) at the bottom of the next page, where $\bar{y}_{k d}=10 \log \bar{y}_{k}, \mu_{k d d}$ is the (area) mean of $\bar{y}_{k d}$, where the subscript $d$ is used to emphasize the fact that the associated random variables are measured in decibels. $\sigma$, the standard deviation, also known as the shadowing spread, has a typical value that lies between 3-12 dB for urban environments [4], [13], [14]. The mean $\mu_{x d}$ is related to the area mean power $\mu_{x}$ by $[12$, ch. 2]

$$
\mu_{x d}=10 \log \left(\eta \mu_{x}\right), \quad \text { with } \eta=\exp \left[-(\sigma \ln 10)^{2} / 200\right] \text {. }
$$


In practice, $\mu_{x}$ can be predicted by a path-loss model suitable for the communication environment under consideration.

When the shadowing effect on a signal reception is taken into account, the outage probability can be evaluated via

$$
\begin{aligned}
P_{\text {out }}= & \int_{-\infty}^{\infty} \cdots \int P_{\text {out } \mid\left(\bar{x}, \bar{y}_{1}, \bar{y}_{2}, \cdots, \bar{y}_{L}\right)} \\
& \times f\left(\bar{x}_{1}, \bar{y}_{1}, \bar{y}_{2}, \cdots, \bar{y}_{L}\right) d \bar{x} d \bar{y}_{1} d \bar{y}_{2} \cdots d \bar{y}_{L}
\end{aligned}
$$

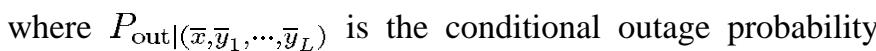
for a given set of local mean desired and undesired powers and can be computed from one of the expressions derived in previous subsections.

1) Multiple i.i.d. Interferers: In this case, all the local mean interference powers $\bar{y}_{1}, \bar{y}_{2}, \cdots, \bar{y}_{L}$ are assumed to be equal to $\bar{y}_{d}$. Let $\tau_{x}=\left(\bar{x}_{d}-\mu_{x d} / \sqrt{2} \sigma\right)$ and $\tau_{y}=\left(\bar{y}_{d}-\mu_{y d} / \sqrt{2} \sigma\right)$ and define the area-averaged CIR $R=\mu_{x} / \mu_{y}=10^{\left(\mu_{x d}-\mu_{y d}\right) / 10}$. The fact that $b=10^{\left(\bar{x}_{d}-\bar{y}_{d}\right) / 10}$ implies $b=R \alpha^{\tau_{x}-\tau_{y}}, c=$ $\left((K+1) \alpha^{-\tau_{x}} / \eta z\right)$ with $\alpha=10^{\sqrt{2} \sigma / 10}$ and $z=\mu_{x} / \gamma_{0}$. The resulting outage probability can be expressed in a Hermitian integration form

$$
\begin{aligned}
P_{\text {out }}= & \frac{1}{\pi} \int_{-\infty}^{\infty} \exp \left(-\tau_{x}^{2}-\tau_{y}^{2}\right) \\
& \times\left[\left.P_{\text {out } \mid(\bar{x}, \bar{y})}\right|_{b=R \alpha^{\left(\tau_{x}-\tau_{y}\right)}, c=\left((K+1) \alpha^{\left.-\tau_{x} / \eta z\right)}\right] d \tau_{x} d \tau_{y}}\right.
\end{aligned}
$$

where $P_{\text {out } \mid(\bar{x}, \bar{y})}$ is that of (13) or (14). Equation (19) can be accurately evaluated by the 2-D Gauss-Hermit quadrature rule.

2) Multiple Interferers with Different Statistics: Substituting (11) or (15) and (16) into (18) and using suitable change of variables and the facts that $b_{k}=10^{\left(\bar{x}_{d}-\bar{y}_{k \cdot d}\right) / 10}, R_{k}=$ $\mu_{x} / \mu_{k}=10^{\left(\mu_{x d}-\mu_{k d}\right) / 10}$, we have

$$
\begin{aligned}
P_{\text {out }}= & \left(\frac{1}{\sqrt{\pi}}\right)^{L+1} \int_{-\infty}^{\infty} \cdots \int_{-\infty} \exp \left(-\tau_{x}^{2}-\tau_{1}^{2}-\cdots-\tau_{L}^{2}\right) \\
& \times\left[\left.P_{\text {out } \mid\left(\bar{x}, \bar{y}_{1}, \cdots, \bar{y}_{L}\right)}\right|_{b_{k}=R_{k} \alpha^{\left(\tau_{x}-u_{k}\right)}, c=\left((K+1) \alpha^{-\tau_{x}} / \eta z\right)}\right] \\
& d \tau_{x} d \tau_{1} \cdots d \tau_{L}
\end{aligned}
$$

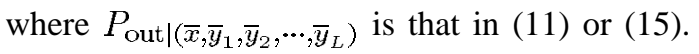

\section{Reuse Distance AND Coverage PREDiction}

\section{A. Path Loss and Reuse Distance}

The normalized cochannel reuse distance $U$, also known as the CCI reduction factor, is defined as

$$
U=\frac{D}{r}
$$

where $r$ is the radius of a cell and $D$ is the distance between the centers (base stations) of the nearest cochannel cells. A practical value of $U$ will be greater than two and one would like $D$ to be as close to this lower bound as possible while satisfying the outage requirement. Let $d$ be the distance between a transmitter and its receiver. The area mean signal power $\mu$, as predicted by the dual-slope path-loss model [19], is given by

$$
\begin{aligned}
& \mu=\kappa \cdot d^{-n_{1}}, \quad d<d_{b k} \\
& \mu=\kappa \cdot d_{b k}^{\left(-n_{1}+n_{2}\right)} \cdot d^{-n_{2}}, \quad d \geq d_{b k}
\end{aligned}
$$

where $\kappa$ is a basic transmission parameter common to the desired signal and the interfering signals, $d_{b k}$ is called the breakpoint distance, and $n_{1}$ and $n_{2}$ represent the power loss exponents before and after the breakpoint, respectively. Measurements in urban environments show [19] that $d_{b k}$ is about $150-300 \mathrm{~m}, n_{1} \approx 2$, and $4 \leq n_{2}<10$. We use $n_{1}=2$ and $n_{2}=4$ in our numerical study.

As a worst case consideration, we assume that the mobile unit is located at the boundary of a cell. In a street microcellular system, the cell radius $r$, which is equal to the maximum distance between a mobile unit and the base station serving it, is often less than $1 \mathrm{~km}$. If $d_{b k} \leq r,(21)$ and (22) lead to

$$
\begin{aligned}
R & =\text { area-averaged CIR } \\
& =\frac{\mu_{x}}{\mu_{y}}=\left(\frac{D-r}{r}\right)^{n_{2}}=(U-1)^{n_{2}} .
\end{aligned}
$$

If $d_{b k}>r$, say $d_{b k}=1.2 r$, then

$$
R=1.2^{\left(n_{1}-n_{2}\right)}(U-1)^{n_{2}}
$$

provided that $U \geq 2.2$. Using the relationship, (23) or (24), one can compute the outage probability as a function of the normalized reuse distance.

\section{B. Random Number of Interferers}

In all cases we have considered so far, the number of interferers is assumed to be fixed. It is possible that the cochannels in some of the nearby frequency reuse cells are not used [4], [20]. The channel status of being used or not can be described by the Bernouli distribution

$$
B_{r}(I)=p_{a}^{I}\left(1-p_{a}\right)^{1-I}, \quad I= \begin{cases}0, & \text { if channel is idle } \\ 1, & \text { if channel is active }\end{cases}
$$

where $0<p_{a}<1$ is the probability that a channel is active. Let $N$ be the number of cochannel interferers and assume that they are statistically i.i.d. Then, the number of active interferers $L$ follows a binomial distribution

$$
P_{i}(L)=\left(\begin{array}{l}
N \\
L
\end{array}\right) p_{a}^{L}\left(1-p_{a}\right)^{N-L}, \quad L=0,1, \cdots, N .
$$

The grade of a mobile radio service is usually specified by the blocking probability $B$ with 0.02 being a typical value at

$$
f\left(\bar{x}_{d}, \bar{y}_{1 d}, \cdots, \bar{y}_{L d}\right)=\left(\frac{1}{\sqrt{2 \pi} \sigma}\right)^{L+1} \times \exp \left[-\frac{\left(\bar{x}_{d}-\mu_{x d}\right)^{2}+\left(\bar{y}_{1 d}-\mu_{1 d}\right)^{2}+\cdots+\left(\bar{y}_{L d}-\mu_{L d}\right)^{2}}{2 \sigma^{2}}\right]
$$




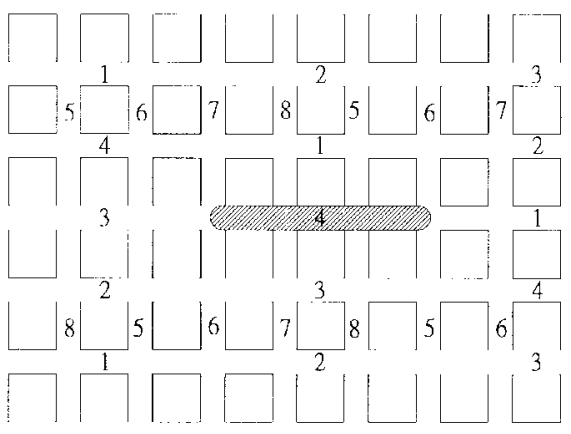

Fig. 1. A street microcellular configuration with a cluster of eight cells. The street blocks are $100(\mathrm{~m}) \times 100(\mathrm{~m})$ and the width of streets is $40 \mathrm{~m}$. Numbers are for positions of cell sites using different frequency bands.

the busy hours [21]. Given the blocking probability and the radio traffic requirement, we can determine the number of base station channels $N_{c}$ in each cell by the use of the Erlang- $B$ formula. If all the available channels are equally likely to be used, then $p_{a}=B^{1 / N_{c}}$ and (25) becomes

$P_{i}(L)=\left(\begin{array}{c}N \\ L\end{array}\right) B^{L / N_{c}}\left(1-B^{1 / N_{c}}\right)^{N-L}, \quad L=0,1, \cdots, N$.

The average outage probability thus becomes

$$
P_{\text {out }}=\sum_{L=0}^{N} P_{\text {out }}(L) P_{i}(L) .
$$

\section{Coverage Prediction}

Although reuse distance can help us in estimating the spectral efficiency and determining the reuse pattern, more detailed information is needed in implementing a cellular system. When one takes the actual street geometry and base station locations into consideration, one of the major design concerns is the cell coverage area, i.e., the area within which mobile stations can be served by the same base station with a prescribed quality and with a probability higher than a given percentage. Coverage area prediction can be roughly derived by estimating the path losses for the locations of interest [22]. However, in a highly variable urban microcellular environment it is not sufficient to just consider the path loss. Outage analysis presented before can be used to estimate the service area for a street microcell by taking into account both signal fading and shadowing effects.

Base stations of a typical urban microcellular communication network are usually located in the streets with their antennas below the urban skyline. The radio coverage depends on the road topology and the dimensions of street blocks. For cities that have grid street patterns and rectangular street blocks, the microcells have regular shapes. Fig. 1 shows a microcellular arrangement with rectangular cells formed by positioning base stations at the middle of blocks [23]. In such a cell configuration, the designed service area of a base station is about one segment, $210 \mathrm{~m}$ on each side of the base station, of a lateral or transverse street.

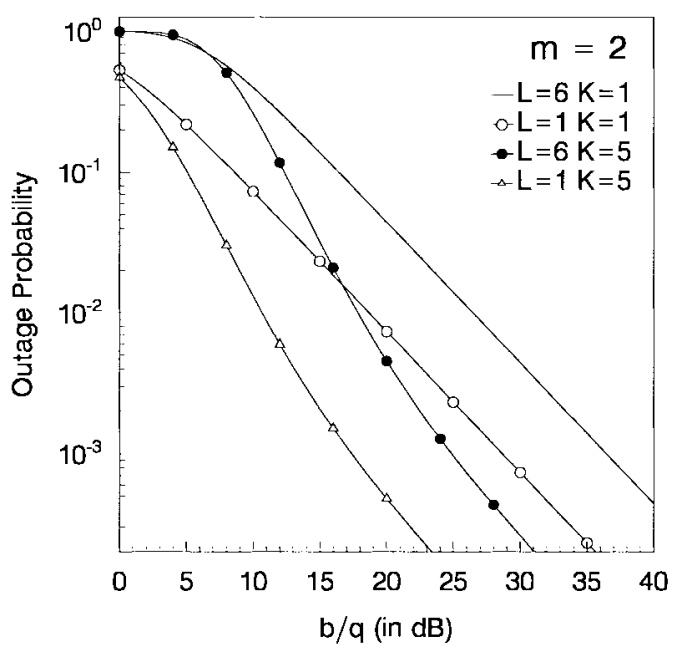

Fig. 2. Outage probability versus $b / q$ in the presence of $L$ i.i.d. interferers.

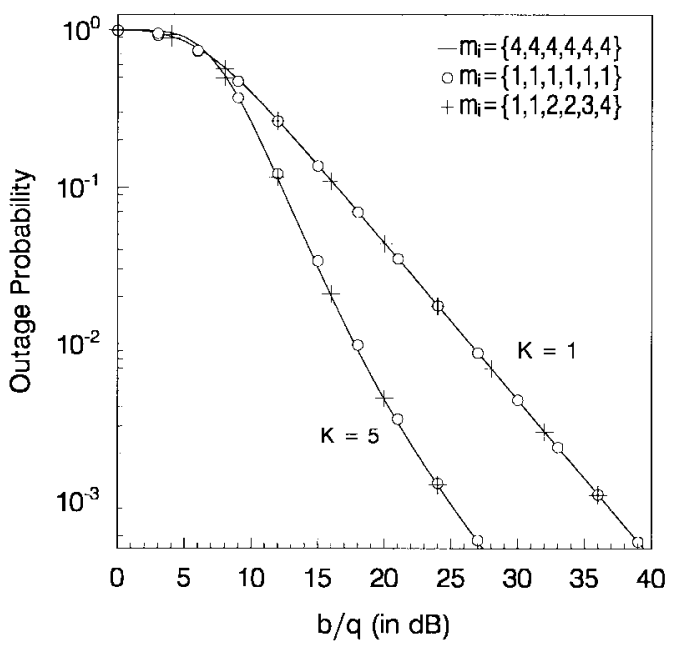

Fig. 3. Outage probability versus $b / q$ for different fading parameter distributions. The number of i.i.d. interferers is $L=6$.

\section{NUMERICAL RESULTS}

Fig. 2 depicts the outage probability as a function of $b / q$ parameterized by $a$ and the number of i.i.d. interferers. For a given outage probability, the fewer the interferers or the larger the Rician factor is, the smaller the required ratio $b / q$ becomes. Shown in Fig. 3 are the outage probabilities caused by the presence of six interferers with three different sets of fading parameters. As one can see from these numerical results, the number of i.i.d. interferers $L$ and the Rician factor $K$ are the dominant factors in determining the outage probability while the fading parameter $m$ has much less effect. The total interference power may be distributed among several interferers or it may be emitted from a single interferer. Fig. 3 examines the outage probability behavior when the same total mean interference power comes from either a single interferer or four independent interferers. The result indicates that the distribution of the interference power has little or no effect on the outage probability.

Fig. 5 shows the outage probability as a function of local mean CIR margin, i.e., $\mathrm{CIR}(\mathrm{dB})-q(\mathrm{~dB})$, when the excess 


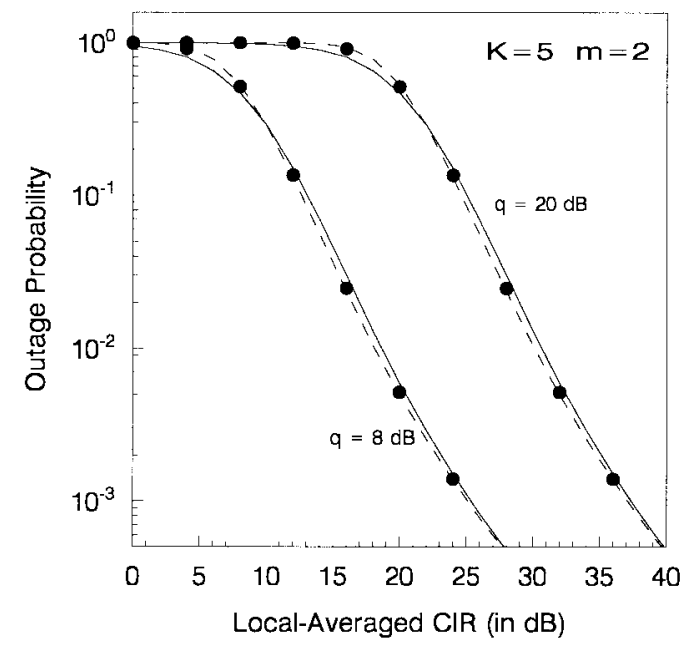

Fig. 4. Comparison of outage probabilities for single interferer and four interferers when the total interference power is fixed. The mean power $\bar{y}$ of a single interferer (solid lines) is equal to the total mean power of four interferers, each with the same mean power $=\bar{y} / 4$ (dashed lines). Dots represent outage probability for cases that the four interferers have different mean powers $\bar{y} / 2, \bar{y} / 3, \bar{y} / 9$, and $\bar{y} / 18$.

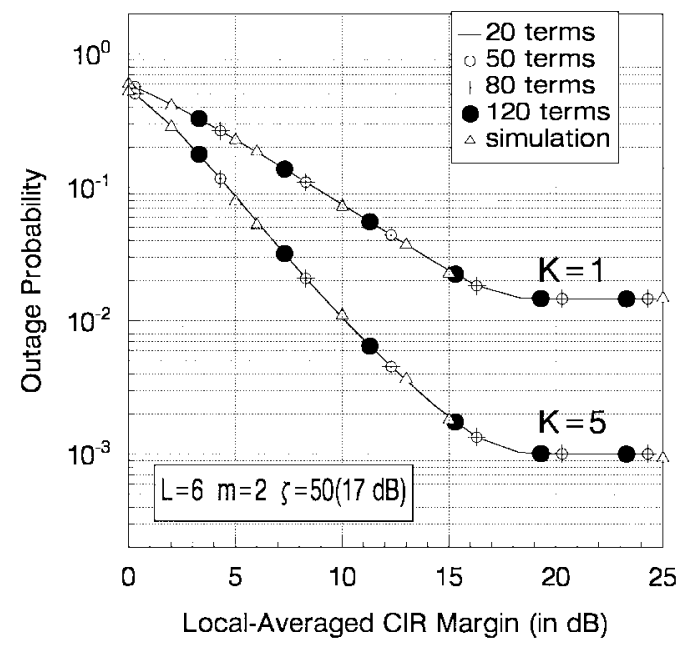

Fig. 5. Comparison of outage probabilities estimated by using simulation and various truncated versions of (13).

signal power $\xi=50(17 \mathrm{~dB})$. It is clear that the minimum signal power requirement sets a lower bound on the outage probability. For a given excess signal power, there is a threshold beyond which the increase of CIR has no effect on reducing the outage probability. This floor value of the outage probability is strongly affected by the Rician factor. In other words, for a noise-limited situation (large CIR) the strength of the desired signal's specular component will dominate the outage probability. Also shown in Fig. 5 are the outage probabilities calculated by using 20,50 , and 80 terms of the series (13) and those estimated by computer simulation. Extensive numerical results have indicated that for those $a, b / q$, and $m$ of interest, the series (11) and (13) converge relatively fast (20-30 terms are required to render an accuracy of order $10^{-8}$ ). Nevertheless, we use 100 terms in all our computations.

The combined effect of fading and shadowing in the presence of six i.i.d. interferers is shown in Fig. 6. To confirm

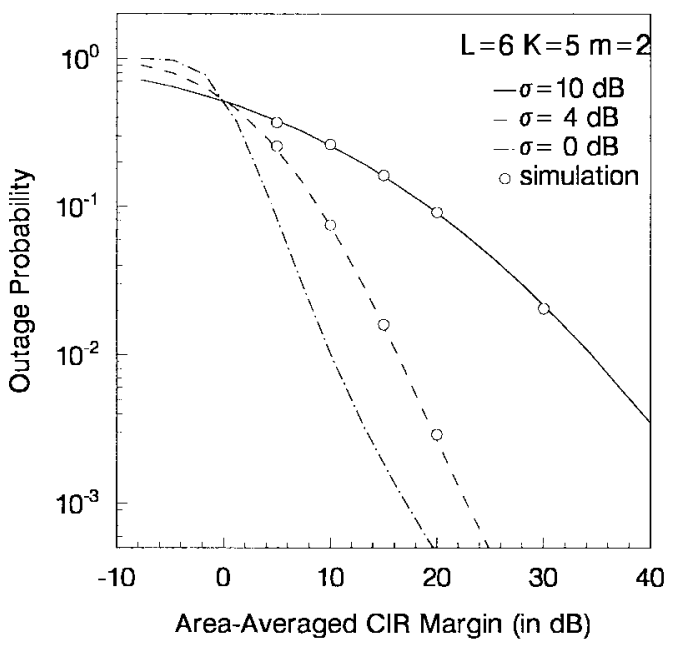

Fig. 6. Outage probability versus mean CIR margin when both fading and shadowing fading are considered.

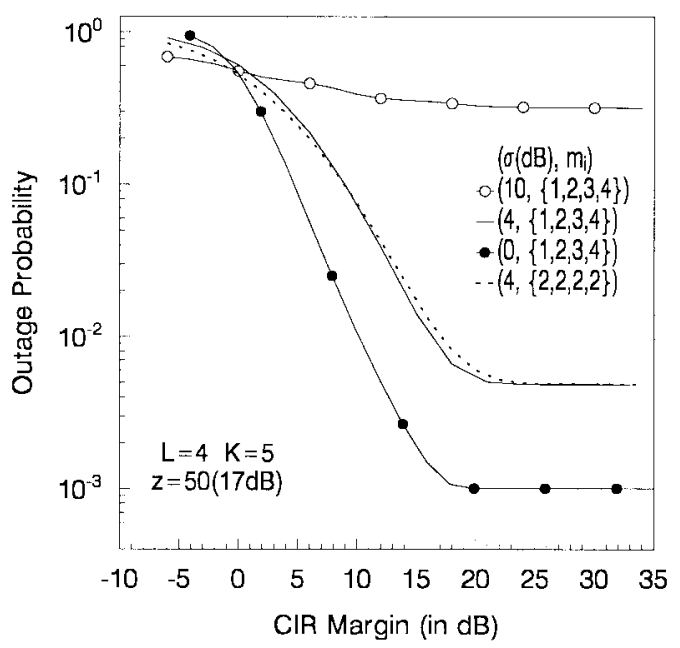

Fig. 7. Outage probability in the presence of four equal-mean-power interferers with identical or different fading parameters. The area mean excess desired signal power $\mu_{x} / \gamma_{0}$ is 50 .

the accuracy of our numerical integration algorithm we have included simulation results in the same figure. We note that for most cases of interest, severer shadowing (larger $\sigma$ ) causes higher outage probability. However, for a very low CIR margin, the trend is reversed. Fig. 7 reveals that the influence of the fading parameters on the outage probability is insignificant. The error floor is determined by the minimum signal power requirement and the shadowing spread. Figs. 8 and 9 show the outage probability as a function of normalized reuse distance in various communication environments, assuming i.i.d. interferers. As expected, the minimum reuse distance is an increasing function of the interferers' number and the system protection ratio, but a decreasing function of the Rician factor $K$.

Fig. 10 illustrates the outage behavior as a function of $U, N_{c}$, and $q$ when the blocking probability $B=0.02$ and the maximum number of interferers is $N=6$. Comparing Fig. 10 with Fig. 9, we conclude that for a given blocking probability, when a larger $N_{c}$ is called for in response to a heavier traffic load, the resulting outage probability increases and approaches 


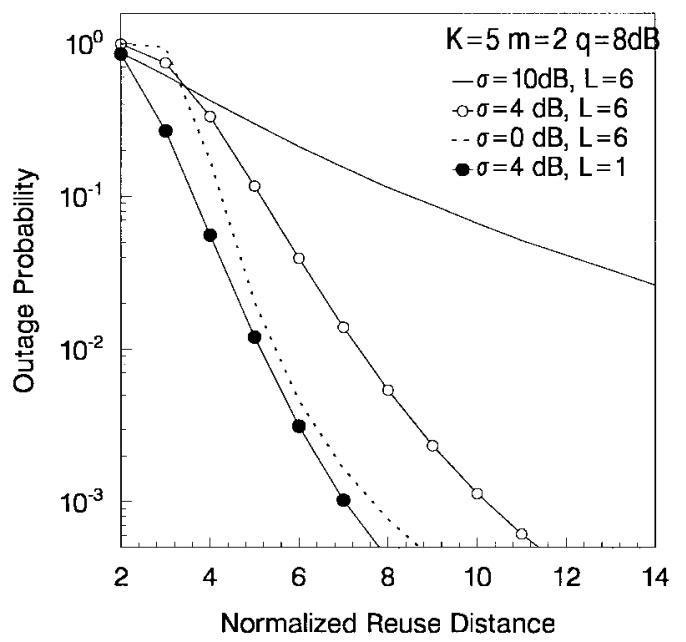

Fig. 8. Outage probability versus normalized reuse distance for various channel states.

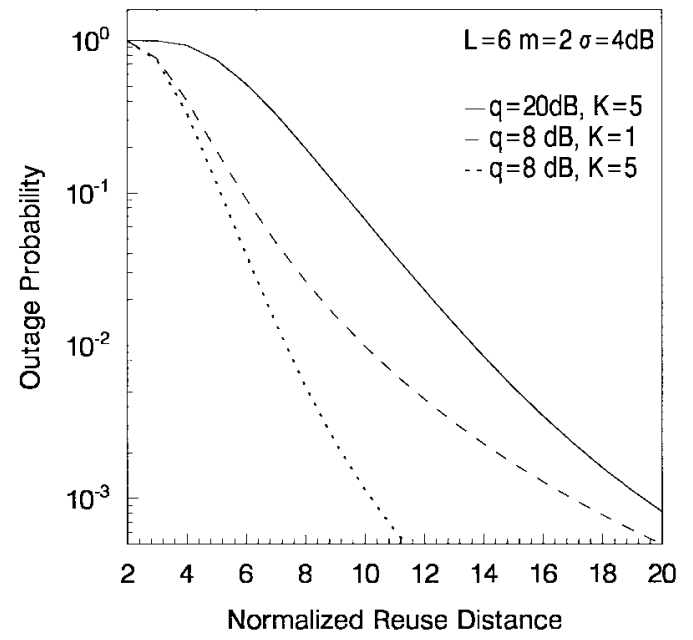

Fig. 9. Outage probability versus normalized reuse distance for various channel states and system protection ratio $q$.

the worst case where all possible interferers are active. This can easily be seen from (26) for if $N_{c} \gg 1$ and $N_{c} \gg N$, we have $B^{L / N_{c}} \approx 1, P_{i}(N) \gg P_{i}(K)$ for all $K<N$. Hence, (27) is dominated by $P_{\text {out }}(N) P_{i}(N)$. The total interference power might be distributed among several interferers or it might come from a single interferer. Fig. 4 examines the outage probability behavior for: 1) a single interferer and 2) for four interferers with the same total mean power. The result indicates that the distribution of the interference power has little or no effect on the outage probability if the interferers are independent.

In estimating the cell coverage, we consider two firsttier LOS CCI's and two nearest non-LOS CCI's in the configuration shown in Fig. 1. For a CCI-limited environment, the probability of outage is a function of area-averaged CIR which can be predicted by an appropriate path-loss model. For a LOS path, the signal power is assumed to obey a dualslope loss law with $n_{1}=2, n_{2}=4$, and $d_{b k}=150 \mathrm{~m}$; for a non-LOS path, the received power follows a singleslope loss with slope equal to 4.5 and an additional corner

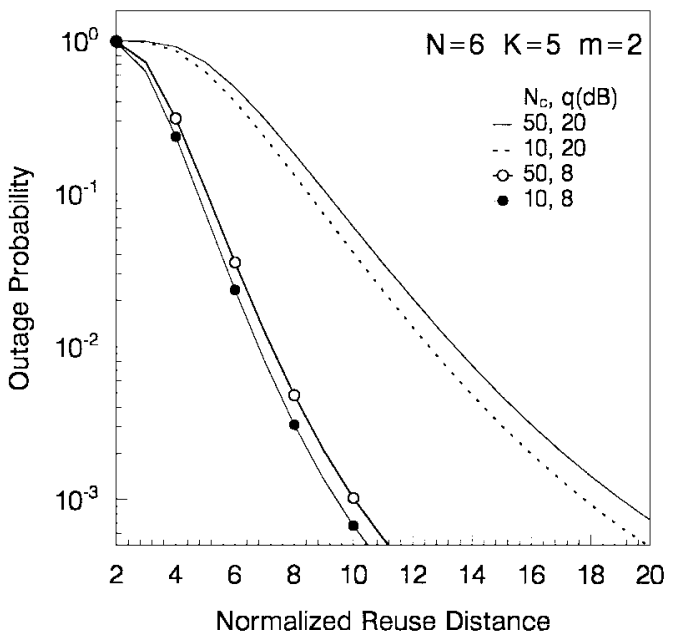

Fig. 10. Outage probabilities versus normalized reuse distance in the presence of random number of interferers. Both fading and shadowing are considered.

loss of $30 \mathrm{~dB}$ [24], [25]. Fig. 11 shows a shadowed region of $P_{\text {out }}(L=4 ; I) \leq 0.01$ and a blank region bounded by the line with $P_{\text {out }}(L=4 ; I) \leq 0.1$. The shadowing spread $\sigma$ is $4 \mathrm{~dB}$, and the system protection ratio is $15 \mathrm{~dB}$. The fading parameters of LOS interferers are set to three to approximate the Rician distributions with Rician factor 5 [2] and those of non-LOS interferers are set to one. The figure shows that almost all of the designed service area is within the region of $P_{\text {out }}(L=4 ; I) \leq 0.01$.

\section{CONCLUSIONS}

A shadowed-Rician/shadowed-Nakagami channel model has been used to study the outage probabilities and coverage predictions of microcellular mobile radio systems. When only short-term fading is considered, we have derived closed-form expressions for the outage probabilities in the presence of multiple independent interferers with identical and different statistics. The influence of lognormal shadowing on both desired and undesired signals is investigated. We also analyzed the impacts of the minimum CIR and minimum signal strength (or, equivalently, minimum CNR) requirements. The outage behavior is studied by varying channel and system parameters like Rician factor, fading parameter, shadowing spread, system CIR protection ratio, and minimum required signal power. Our numerical investigation has concluded that the outage probabilities are dominated by the Rician factor and are insensitive to the fading parameter.

Using a dual-slope power (path) loss law, we determine adequate reuse distances that ensure a given worst case outage probability. Numerical results tell us that the average outage probability that takes into account the fact that the number of interferers is random tends to converge to the worst case performance if the number of the base station channels is large. Finally, we demonstrate another useful application of our analysis in cell planning: estimating the coverage contours for a given microcellular configuration. Such an estimation enables us to accurately predict the area within which a satisfactory 


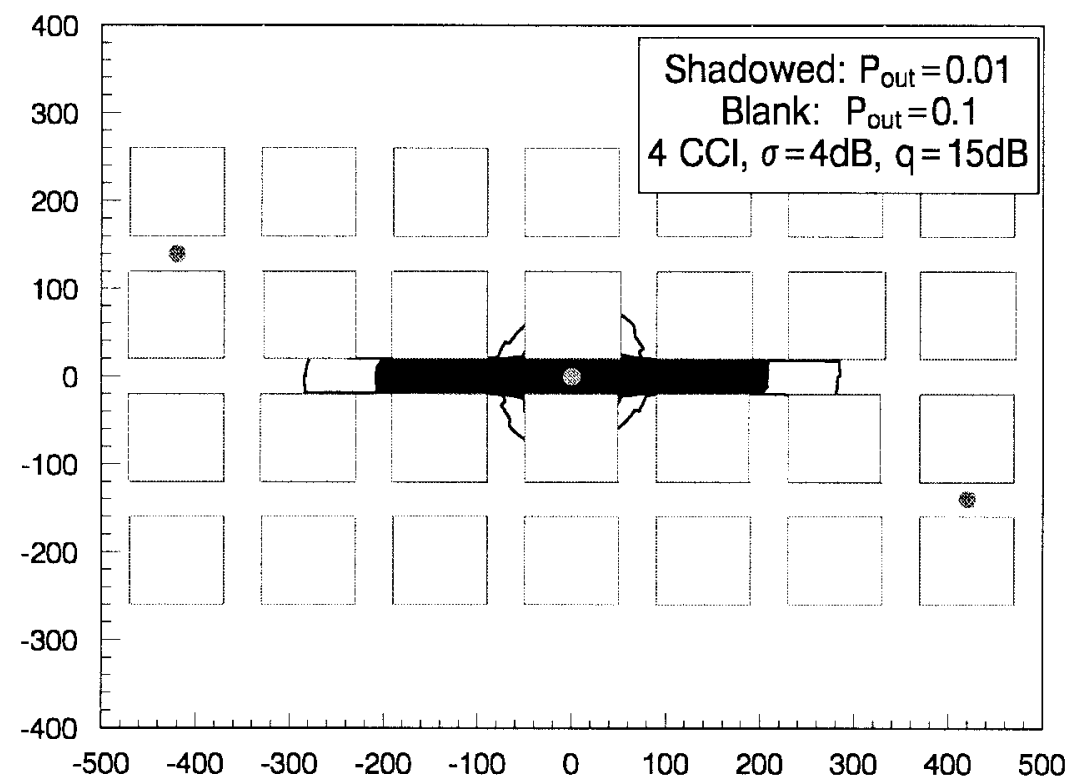

Fig. 11. Constant outage probability contours in a street microcellular environment. The dots denote the positions of cochannel base stations: the desired one is at $(0,0)$, two non-LOS stations are at $(420,-140)$ and $(-420,140)$, and two LOS stations (not shown) are at $(1680,0)$ and $(-1680,0)$.

reception quality can be anticipated with a probability higher than a prespecified value.

\section{APPENDIX I}

Outage Probabilities Due to CCI AND Thermal Noise

\section{A. Multiple i.i.d. Interferers}

Substituting (3) and (9) with $m_{t}=m L$ into (10), we obtain

$$
\begin{aligned}
P_{\text {out } \mid \bar{x}, \bar{y}}(L ; I, N) & \\
= & 1-\int_{\gamma_{0}}^{\infty} \frac{K+1}{\bar{x}} \\
\times & \exp \left(-K-\frac{K+1}{\bar{x}} x\right) I_{0}\left(\frac{\sqrt{4 K(K+1)}}{\bar{x}} x\right) \\
& \cdot\left(\frac{m}{\bar{y}}\right)^{m L} \frac{1}{\Gamma(m L)} \int_{0}^{x / q} y^{m L-1} \\
& \quad \exp \left(-\frac{m}{\bar{y}} y\right) d y d x .
\end{aligned}
$$

Invoking the identities of [18, eqs. (3.381.1) and (8.352.1)], we rewrite the double integral on the right-hand side of (I.1) as

$$
\begin{aligned}
& \frac{K+1}{\bar{x}} e^{-K}\left[\int_{\gamma_{0}}^{\infty} e^{(-x(K+1) / \bar{x})} I_{0}\left(\sqrt{\frac{4 K(K+1) x}{\bar{x}}}\right) d x\right. \\
& \quad-\sum_{k=0}^{m L-1} \frac{\left(\frac{m}{q \bar{y}}\right)^{k}}{k !} \int_{\gamma_{0}}^{\infty} x^{k} \exp \left(-\frac{K+1}{\bar{x}} x-\frac{m x}{\bar{y} q}\right) \\
&\left.\times I_{0}\left(\sqrt{\frac{4 K(K+1) x}{\bar{x}}}\right) d x\right] .
\end{aligned}
$$

Using [18, eq. (8.447.1)]

$$
I_{0}\left(\sqrt{\frac{4 K(K+1) x}{\bar{x}}}\right)=\sum_{h=0}^{\infty} \frac{1}{h ! h !}\left(\frac{K(K+1)}{\bar{x}} x\right)^{h}
$$

and the identity [18, eq. (3.381.3)]

$$
\int_{u}^{\infty} x^{\nu-1} e^{-\mu x} d x=\mu^{-\nu}(\nu-1) ! e^{(-\mu u)} \sum_{m=0}^{\nu-1} \frac{(\mu u)^{m}}{m !}
$$

and invoking the definitions $t=(K+1) x / \bar{x}, c=\gamma_{0}(K+$ $1) / \bar{x}, b=\bar{x} / \bar{y}$, and $w=(m b / q(K+1))$ yields (13).

\section{B. Interferers with Different Statistics}

Substituting (3) and (7) into (10), we obtain

$$
\begin{aligned}
P_{\text {out } \mid \bar{x}, \bar{y}_{1}, \bar{y}_{2}, \cdots, \bar{y}_{L}}(L ; I, N) \\
=1-\int_{\gamma_{0}}^{\infty} \frac{K+1}{\bar{x}} \\
\quad \times \exp \left(-K-\frac{(K+1) x}{\bar{x}}\right) I_{0}\left(\sqrt{\frac{4 K(K+1) x}{\bar{x}}}\right) \\
\quad \times\left[\prod_{k=1}^{L}\left(\frac{m_{k}}{\bar{y}_{k}}\right)^{m_{k}}\right] \sum_{k=1}^{L} \sum_{i=1}^{m_{k}} \frac{z_{k}^{i-1}}{\left(m_{k}-i\right) !(i-1) !} \\
\quad \times \int_{0}^{x / q} y^{m_{k}-i} \exp \left(-\frac{m_{k}}{\bar{y}_{k}} y\right) d y d x .
\end{aligned}
$$

The integral with respect to $y$ has the form of the incomplete Gamma function. Substituting a complementary representation 
of (I.4) into (I.5) gives

$$
\begin{aligned}
P_{\text {out } \mid \bar{x}, \bar{y}_{1}, \bar{y}_{2}, \cdots, \bar{y}_{L}}(L ; I, N) & \\
= & 1-\frac{K+1}{\bar{x}} e^{-K}\left[\prod_{k=1}^{L} w_{k}^{m_{k}}\right] \\
& \cdot \sum_{k=1}^{L} \sum_{i=1}^{m_{k}} \frac{D_{k}^{i-1}}{(i-1) !} w_{k}^{-m_{k}+i-1} \\
& \cdot\left[\int_{\gamma_{0}}^{\infty} \exp \left(-\frac{K+1}{\bar{x}} x\right)\right. \\
& \cdot I_{0}\left(\sqrt{\frac{4 K(K+1) x}{\bar{x}}}\right) d x-\sum_{j=0}^{m_{k}-i} \frac{1}{j !}\left(\frac{m_{k} b_{k}}{\bar{x} q}\right)^{j} \\
& \cdot \int_{\gamma_{0}}^{\infty} x^{j} e^{-x\left((K+1) / \bar{x}+\left(m_{k}, b_{k} / \bar{x} q\right)\right)} \\
& \left.\cdot I_{0}\left(\sqrt{\frac{4 K(K+1) x}{\bar{x}}}\right) d x\right] .
\end{aligned}
$$

The integrals within the bracket have forms similar to those in (I.2). Equation (11) can then be obtained by following an analogous procedure.

\section{APPENDIX II}

\section{Outage Probabilities in a CCI-Limited ENVIRONMENT}

Substituting (3) and (9) with $m_{t}=m L$ and $\gamma_{0}=0$ into (10) and invoking (I.4) and the identity [18, eq. (6.643.2)]

$$
\begin{aligned}
\int_{0}^{\infty} & x^{u-(1 / 2)} e^{-\alpha x} I_{2 v}(2 \beta \sqrt{x}) d x \\
= & \frac{\Gamma\left(u+v+\frac{1}{2}\right)}{\Gamma(2 v+1)} \beta^{-1} \exp \left(\frac{\beta^{2}}{2 \alpha}\right) \alpha^{-u} M_{-u, v}\left(\frac{\beta^{2}}{\alpha}\right)
\end{aligned}
$$

where $M_{\lambda, \mu}(z)$ is the Whittaker function defined by [18, eq. (9.220.2)]

$$
M_{\lambda, \mu}(z)=z^{\mu+(1 / 2)} e^{-(z / 2)} \sum_{n=0}^{\infty} \frac{\left(\mu-\lambda+\frac{1}{2}\right)_{n}}{(2 \mu+1)_{n}} \frac{z^{n}}{n !}
$$

and $(x)_{n}=\Gamma(x+n) / \Gamma(x)$, we obtain

$$
\begin{aligned}
& P_{\text {out } \mid \bar{x}, \bar{y}}(L ; I) \\
& =e^{-K} \sum_{k=0}^{m L-1} \frac{1}{1+w}\left(\frac{w}{1+w}\right)^{k} * \\
& \times \sum_{i=0}^{\infty} \frac{(k+i) !}{k ! i ! i !}\left(\frac{K}{1+w}\right)^{i}
\end{aligned}
$$

with $w=(m b / q(K+1))$ and $b=\bar{x} / \bar{y}$. The infinite series on the right-hand side of (II.3) can be simplified according to [15]

$$
\begin{aligned}
\sum_{i=0}^{\infty} & \frac{(k+i) !}{k ! i ! i !}\left(\frac{K}{1+w}\right)^{i} \\
& =\exp \left(\frac{K}{1+w}\right) \sum_{j=0}^{k} \frac{k !\left(\frac{K}{1+w}\right)^{j}}{(k-j) ! j ! j !}
\end{aligned}
$$

where $k=0,1,2, \cdots$. Combining (II.3) and (II.4), we then obtain (14).

Equation (15) can be obtained by: 1) substituting $\gamma_{0}=0$, (3), and (9) into (10); 2) using (I.4) to perform the integration with respect to $y ; 3)$ applying the identity

$$
\left[\prod_{k=1}^{L}\left(\frac{m_{k}}{\bar{y}_{k}}\right)^{m_{k}}\right]=\left[\prod_{k=1}^{L} w_{k}^{m_{k}}\right]\left[\prod_{k=1}^{L}\left(\frac{\bar{x}}{q(K+1)}\right)^{-m_{k}}\right]
$$

where

$$
w_{k}=\frac{m_{k}}{\bar{y}_{k}} \frac{\bar{x}}{q(K+1)}
$$

and

$$
z_{k}^{i-1}=D_{k}^{i-1}\left(\frac{\bar{x}}{q(K+1)}\right)^{i-1}\left[\prod_{\substack{\nu=1 \\ \nu \neq k}}\left(\frac{\bar{x}}{q(K+1)}\right)^{m_{\nu}}\right]
$$

and then 4) performing the integration with respect to $x$.

\section{REFERENCES}

[1] R. J. C. Bultitude and G. K. Bedal, "Propagation characteristics on microcellular urban mobile radio channels at $910 \mathrm{MHz}, "$ IEEE J. Select. Areas Commun., vol. 7, pp. 31-39, Jan. 1989.

[2] M. Nakagami, "The $m$-distribution-A general formula of intensity distribution of fading," in Statistical Methods in Radio Wave Propagation, W. C. Hoffman, Ed. London, U.K: Pergamon, 1960.

[3] K. Pahlavan and A. H. Levesque, Wireless Information Networks. New York: Wiley 1995.

[4] A. A. Abu-Dayya and N. C. Beaulieu, "Outage probabilities of cellular mobile radio systems with multiple Nakagami interferers," IEEE Trans. Veh. Technol., vol. 40, 4, pp. 757-768, Nov. 1991.

[5] Y.-D. Yao and A. U. H. Sheikh, "Investigations into cochannel interference in microcellular mobile radio systems," IEEE Trans. Veh. Technol., vol. 41, pp. 114-123, May. 1992.

[6] _ "Outage probability analysis for microcell mobile radio systems with cochannel interferers in Rician/Rayleigh fading environment," Electron. Lett., vol. 26, no. 13, pp. 864-866, 1990.

[7] R. H. Muammar, "Co-channel interference in microcellular mobile radio system," in Proc. 41st IEEE Veh. Technol. Conf., 1991, pp. 198-203.

[8] R. Prasad, A. Kegel, and M. B. Loog, "Cochannel interference probability for picocellular system with multiple Rician faded interferers," Electron. Lett., vol. 28, no. 24, pp. 2225-2226, 1992.

[9] S. Pupolin, L. Tomba, and R. Corvaja, "Outage probability in personal communication systems in the presence of Rician fading," in Proc. ICC'94, pp. 1526-1530.

[10] J. R. Haug and D. R. Ucci, "Outage probability of microcellular radio systems in a Rayleigh/Rician fading environment," in Proc. ICC'92, pp. 312.4.1-312.4.5.

[11] W. C. Jakes, Ed., Microwave Mobile Communications. New York: Wiley, 1974

[12] W. C. Y. Lee, Mobile Communications Engineering. New York: McGraw-Hill, 1993.

[13] P. E. Mogensen et al., "Urban area radio propagation measurements at 955 and $1845 \mathrm{MHz}$ for small and micro cells," in GLOBECOM '91, pp. 1297-1302.

[14] J. E. Berg, R. Bownds, and F. Lotes, "Path loss and fading models for microcells at $900 \mathrm{MHz}$," in Proc. IEEE Veh. Technol. Conf., 1992, pp. 666-671.

[15] W.-C. Kao, "Outage analysis and coverage prediction for microcellular systems," Master's thesis, National Chiao Tung Univ., Taiwan, R.O.C. June 1996.

[16] K. W. Sowerby and A. G. Williamson, "Outage probability calculations for multiple cochannel interferers in cellular mobile radio systems," Proc. Inst. Elect. Eng., vol. 135, pt. F, pp. 208-215, June 1988.

[17] C. Tellambura and V. K. Bhargava, "Outage probability analysis for cellular mobile radio systems subject to Nakagami fading and shadowing," IEICE Trans. Commun., vol. E78-B, no. 10, pp. 1416-1423, Oct. 1995. 
[18] I. Gradshteyn and I. Ryzhik, Table of Integrals, Series, and Products. New York: Academic, 1980.

[19] A. M. D. Turkmani and A. A. Arowojolu, "Microcellular propagation for PCN networks-A review," in Proc. 3rd Int. Conf. Universal Personal Commun., 1994, pp. 171-177.

[20] R. Muammar and S. C. Gupta, "Co-channel interference in high capacity mobile radio systems," IEEE Trans. Commun., vol. COM-30, pp. 1973-1982, Aug. 1982.

[21] W. C. Y. Lee, Mobile Cellular Telecommunications Systems. New York: McGraw-Hill, 1990

[22] L. R. Maciel and H. L. Bertoni, "Cell shape for microcellular systems in residential and commercial environments," IEEE Trans. Veh. Technol., vol. 43, pp. 270-278, May 1994.

[23] H. Persson, "Microcellular structures and their performance," in Proc. IEEE Veh. Technol. Conf., 1992, pp. 413-418.

[24] J. B. Andersen, T. S. Rappaport, and S. Yoshida, "Propagation measurements and models for wireless communications channels," IEEE Commun. Mag., vol. 33, pp. 42-49, Jan. 1995.

[25] H. L. Bertoni et al., "UHF propagation prediction for wireless personal communications," IEEE Proc., vol. 82, pp. 1333-1358, Sept. 1994.

Jen-Cheng Lin received the B.S. degree in electronics engineering from Chung Yuan Christian University, Chungli, Taiwan, R.O.C., in 1979 and the M.S. degree in computer science and information engineering and the $\mathrm{Ph} . \mathrm{D}$. degree in electronics engineering in 1990 and 1995, respectively, from National Chiao Tung University, Hsinchu, Taiwan.

He joined ERSO/ITRI, Hsinchu, in 1983, where he primarily worked on the development of device drivers as a Project Leader. In 1986, he was with New Development Corporation, Hsinchu. In 1987, he was with Link Communications, Inc., Hsinchu, where he worked on the development of a statistical multiplexer and VSAT system as a Project Manager. From November 1990 to March 1993, he served as a Technical Consultant to the Microelectronics Technology, Inc., Hsinchu, on a maritime communications system and personal communications system. He is currently an Associate Researcher at the National Space Program Office, National Science Council, Executive Yuan, Hsinchu. His current research interests include wireless communication networks and satellite communication systems.
Wen-Chang Kao received the B.S. and M.S. degrees in communication engineering from the National Chiao Tung University, Hsinchu, Taiwan, R.O.C., in 1994 and 1996, respectively.

$\mathrm{He}$ is currently an Assistant Researcher at the National Space Program Office, National Science Council, Executive Yuan, Hsinchu. His current research interests include satellite communication and wireless communication systems.

Yu T. Su (S'81-M'83) received the B.S.E.E. degree from Tatung Institute of Technology, Taiwan, R.O.C., in 1974 and the M.S. and Ph.D. degrees from the University of Southern California, Los Angeles, in 1983.

From 1983 to 1989, he was with LinCom Corporation, Los Angles. Since September 1989, he has been a Faculty Member of the Department of Communication Engineering and the Microelectronic and Information Systems Research Center, National Chiao Tung University, Hsinchu, Taiwan. His present research interests include communication theory and statistical signal processing.

Tsern-Huei Lee (S'86-M'87) received the B.S. degree from National Taiwan University, Taipei, Taiwan, R.O.C., in 1981, the M.S. degree from the University of California, Santa Barbara, in 1984, and the Ph.D. degree from the University of Southern California, Los Angeles, in 1987, all in electrical engineering.

Since 1987, he has been a Faculty Member of National Chiao Tung University, Hsinchu, Taiwan, where he is a Professor in the Department of Communication Engineering and a Member of the Center for Telecommunications Research. His current research interests are in wireless communications, communication protocols, broad-band switching networks, and network flow control.

Dr. Lee received an Outstanding Paper Award from the Institute of Chinese Engineers in 1991. 